\title{
Ex vivo confocal microscopy: an emerging technique in dermatology
}

\author{
Elisa Cinotti ${ }^{1}$, Jean Luc Perrot ${ }^{2}$, Bruno Labeille ${ }^{2}$, Frédéric Cambazard ${ }^{2}$, Pietro Rubegni ${ }^{1}$
}

1 Department of Medical, Surgical and Neurological Science, Dermatology Section, University of Siena, S. Maria alle Scotte Hospital, Siena,
Italy
2 Department of Dermatology, University Hospital of Saint-Étienne, Saint-Étienne, France

Key words: confocal microscopy, dermatology, ex vivo, fluorescence, skin, reflectance

Citation: Cinotti E, Perrot JL, Labeille B, Cambazard F, Rubegni P. Ex vivo confocal microscopy: an emerging technique in dermatology. Dermatol Pract Concept. 2018;8(2):109-119. DOI: https://doi.org/10.5826/dpc.0802a08

Received: July 3, 2017; Accepted: January 18, 2018; Published: April 30, 2018

Copyright: (C2018 Cinotti et al. This is an open-access article distributed under the terms of the Creative Commons Attribution License, which permits unrestricted use, distribution, and reproduction in any medium, provided the original author and source are credited.

Funding: None.

Competing interests: The authors have no conflicts of interest to disclose.

All authors have contributed significantly to this publication.

Corresponding author: Elisa Cinotti, PhD. Department of Medical, Surgical and Neurological Science, Dermatology Section, University of Siena, S. Maria alle Scotte Hospital, Viale Bracci 16, 53100 Siena, Italy. Tel: 0039-0577 585428. Fax: 0039 0577585484. Email: elisacinotti@gmail.com

ABSTRACT This review aims to give an overview of the current available applications of ex vivo confocal microscopy (EVCM) in dermatology. EVCM is a relatively new imaging technique that allows microscopic examination of freshly excised unfixed tissue. It enables a rapid examination of the skin sample directly in the surgery room and thus represents an alternative to the intraoperative micrographic control of the surgical margins of cutaneous tumors by standard microscopic examination on cryopreserved sections during Mohs surgery. Although this technique has mainly been developed for the margin's control of basal cell carcinoma, many other skin tumors have been studied, including melanoma. Use of EVCM is continuing to evolve, and many possible applications are under investigation, such as the study of nails and hair diseases and the diagnosis of skin infections.

\section{Introduction}

Ex vivo confocal microscopy (EVCM) is a an emerging imaging technique that allows real-time microscopic examination of freshly excised cutaneous tissue that eliminates the need for standard embedding, processing, and sectioning that is necessary for conventional histology [1]. It enables a rapid examination of an entire skin sample directly in the surgical room. It has been mainly applied to the perioperative analysis of the surgical margins of basal cell carcinoma (BCC) during micrographic Mohs surgery (MMS). Notably, EVCM does not alter the tissue and does not prevent subsequent histopathological examination.

Many possible applications are under investigation, such as the study of skin tumors other than BCC, the evaluation of skin appendages, and the diagnosis of skin infections. We aim to give an overview of the current available applications for this new device. The review was performed using the PubMed database. Search terms employed were "ex vivo," "reflectance confocal microscopy," "confocal laser scanning microscopy," "fluorescence confocal microscopy," "skin," "dermatology," and "surgery." 


\section{Ex vivo Confocal Microscopy Examination}

\section{Microscope}

Only one device is commercially available (VivaScope 2500(r), new version of VivaScope 2000, produced by Caliber, New York, USA and distributed in Europe by MAVIG GmbH, Munich, Germany). The ex vivo confocal microscope works in reflectance mode (laser wavelength of $830 \mathrm{~nm}$ ) or fluorescence mode (laser wavelength of $488 \mathrm{~nm}$ or $658 \mathrm{~nm}$ ).

\section{Processing}

In reflectance mode no staining is required. However, aluminum chloride, acetic acid, or citric acid can be used to enhance the reflectance of nuclei [2-4]. When using the fluorescence mode, the entire surgical specimen is dipped in a solution of a fluorescent agent (e.g., acridine orange, methylene blue, fluorescein, Nile blue, or Patent Blue V) for 10-20 seconds and rinsed in physiological saline, acetic acid or phosphate buffered saline in order to remove the excess of the fluorescent agent. The most used fluorescent agent is acridine orange that targets nuclear DNA [2]. Several studies showed that acridine orange immersion does not affect subsequent frozen sections and formalin-fixed histopathology quality [5-7].

Cutting in thin slices is not necessary. The skin specimen can be observed entirely, or the tumor's margins can be vertically cut as for conventional histology [8].

In order to prevent sample deformation and movement during the examination, the skin sample should be mounted between two thick microscopy glass slides attached together by a small amount of silicon glue or modeling clay [9]. To facilitate the contact of the outer surface of the specimen with the glass slide facing the microscope objective, aqueous gel (e.g., Carbomer gel, Gel Larmes Thea Laboratories, Clermont-Ferrand, France) or silicone can be applied [9]. A "tissue press" can be used to better flatten the sample [9] and avoid to artifacts that result from the inhomogeneous contact between the sample's surface and the glass slide in the case of a specimen with a inhomogeneous thickness. The skin sample is then settled on the microscope stage.

\section{Ex vivo Confocal Microscopy Images}

The microscope produces horizontal images of $750 \times 750$ $\mu \mathrm{m}$ of the different layers of the skin up to a thickness of 200 $\mu \mathrm{m}$. Single images are automatically stitched together into a reconstructed mosaic image to a maximum size of $20 \times 20 \mathrm{~mm}$ (12 x12 mm for the previous generation of the device, the one that is most utilized). The depth of observation is manually adjusted. The acquisition time for a single image of $750 \times 750$ $\mu \mathrm{m}$ is 0.68 seconds $\left(2\right.$ minutes $\left./ \mathrm{cm}^{2}\right)$ for the third generation of the device [8]. According to the manufacturer's data, the lateral and axial spatial resolutions are $1 \mu \mathrm{m}$ and $3 \mu \mathrm{m}$ respectively.
In reflectance mode, the images appear in grayscale with dark hypo reflective structures (black and dark gray) and bright hyper reflective structures (white and light gray) with an identical appearance to in vivo reflectance confocal microscopy (Figure 1). In the fluorescence mode, the nonfluorescent structures are dark (black and dark gray) and the fluorescent structures are white (Figure 2). Using acridine orange, the nuclei of the cells are fluorescently stained in white with an increase in contrast of keratinocytes, hair follicle epithelium, sebaceous and eccrine glands, fibroblasts (Figure 2), and tumor cells (Figure 3) relative to the surrounding tissue.

Different from in vivo reflectance confocal microscopy, muscle and adipose tissue can be seen, given the opportunity to observe the sample from its lateral sides (Figures 1,2) and its bottom. It should be noted that fat tissue is usually modified by fixation during classical histology, whereas fat remains intact under EVCM.

Interestingly, the same specimen can be observed both under the reflectance and fluorescence mode and the combination of the two techniques can give complementary information on the architectural and cellular features.

\section{Applications}

EVCM has been shown to be a reliable diagnostic method with good histological correspondence for both normal and pathological skin $[5,6,10]$. It has been developed as an alternative to the optical microscopy examination of frozen sections stained with hematoxylin and eosin (H\&E) for the intraoperative control of surgical margins of cutaneous tumors during MMS [4,7,10-13].

EVCM has also been used for intraoperative examination of nails $[14,15]$ and can be employed for the evaluation of hair morphology [16] (Figure 4) and disease of the mucosa $[17,18]$. Mosaicing may offer a means to perform rapid histology at the bedside. Several future applications are possible, such as the identification of fillers in the skin [19] and the diagnosis of inflammatory and infectious diseases [20-22].

EVCM cannot replace conventional histological examination because the cytological and architectural details cannot always be clearly identified, and to date, there is no specific staining that allows us to distinguish between different cell types. However, EVCM could be useful in some conditions in which the cellular and architectural abnormalities are more characteristic (Figure 5). Recently, EVCM has extended to other disciplines that might require extemporaneous examination of tumors, such as brain, breast and thyroid tumors [6,23-25]. A possible barrier to more widespread use of EVCM is that the mosaics are based on a single mode of grayscale contrast and appear black and white, whereas histology is based on two stains (hematoxylin for nuclei and 


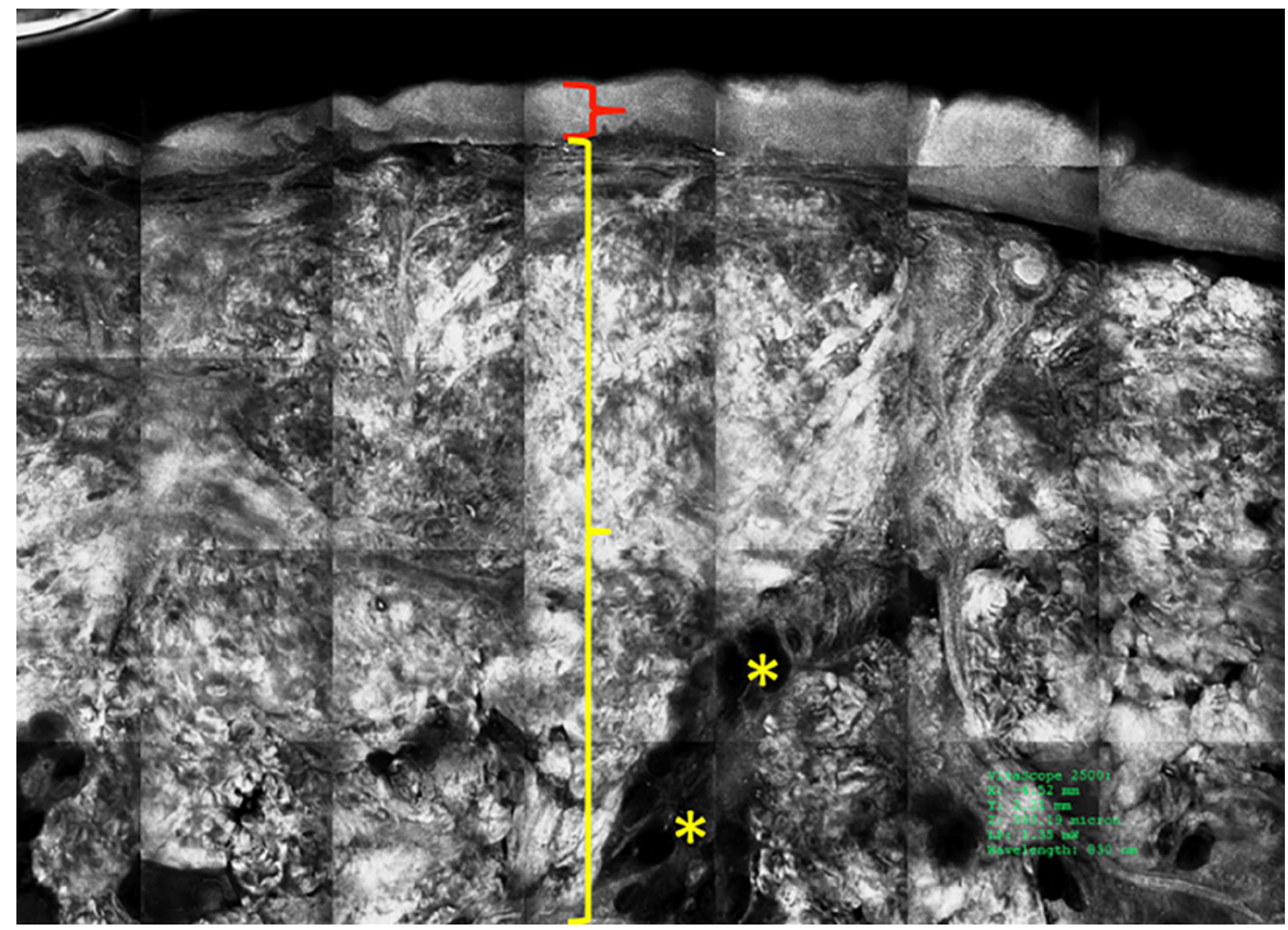

Figure 1. Normal skin under the ex vivo confocal microscope in the reflectance mode. Epidermis (red bracket), dermis (yellow bracket), and fat tissue (yellow asterisks) are visible in a vertical section. [Copyright: (O2018 Cinotti et al.]

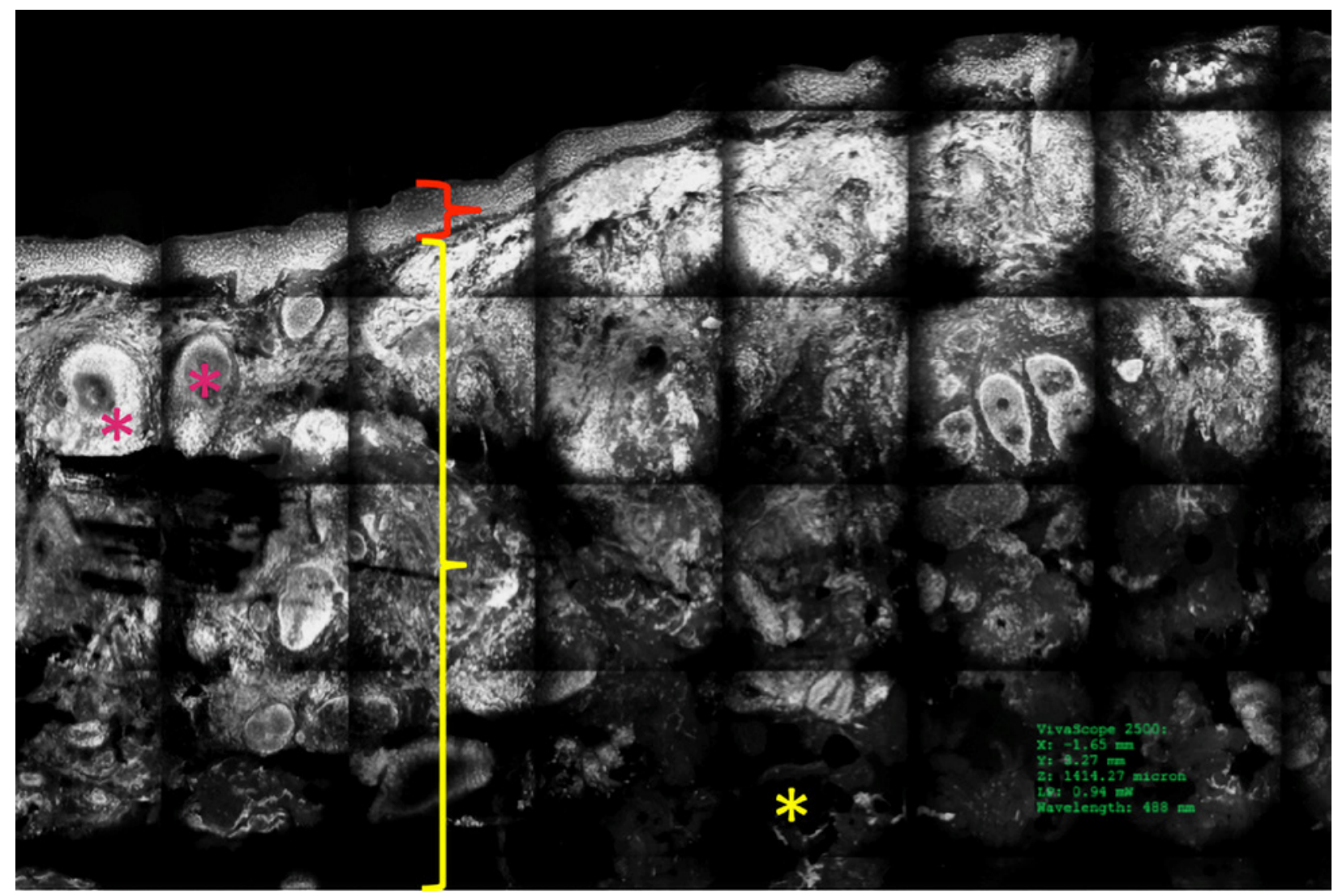

Figure 2. Normal skin under ex vivo confocal microscope in fluorescence mode with acridine orange. Epidermis (red bracket), dermis (yellow bracket), fat tissue (yellow asterisk), and adnexa (pink asterisks) are visible in a vertical section. [Copyright: @2018 Cinotti et al.] 


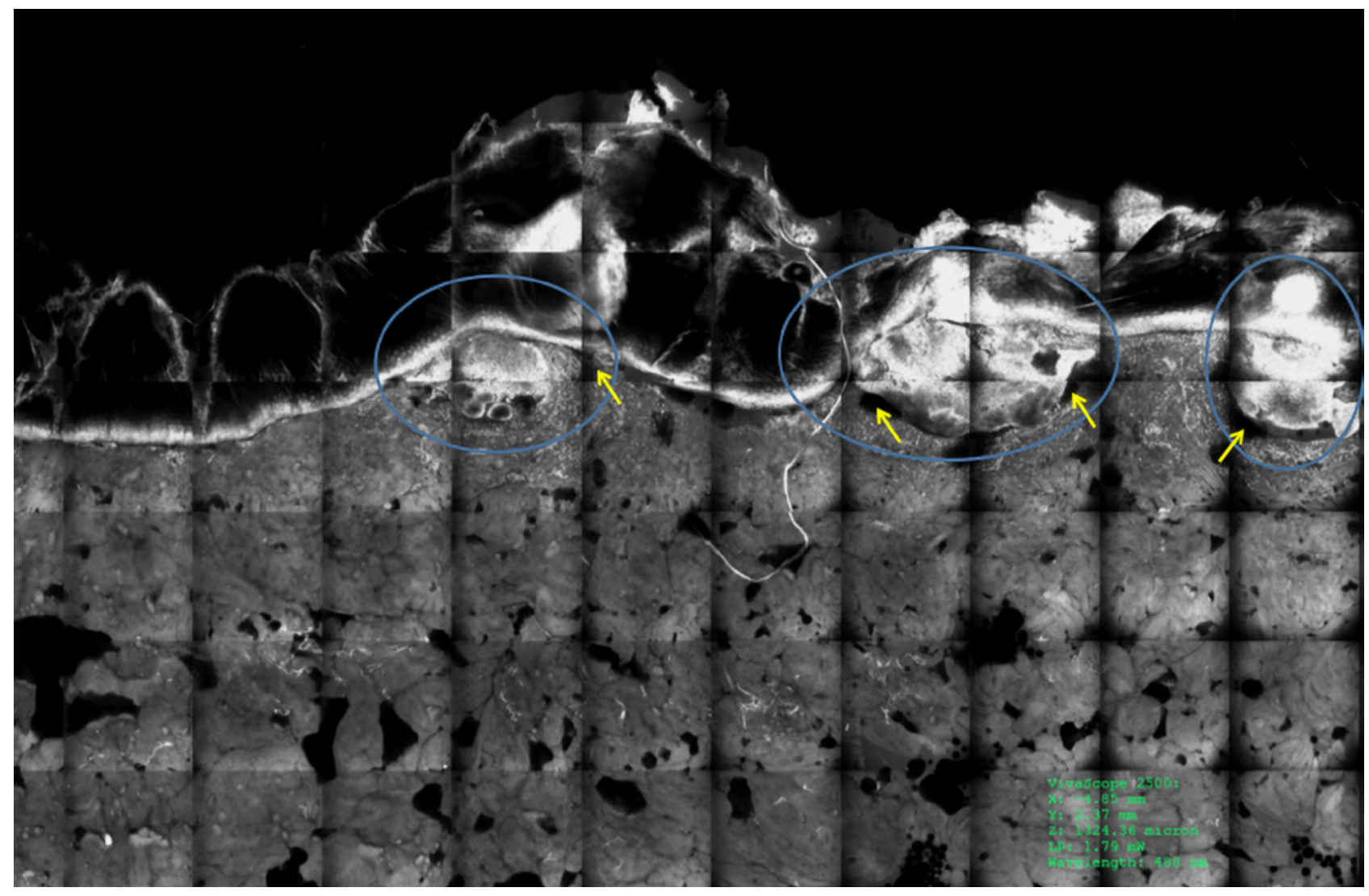

Figure 3. Superficial basal cell carcinoma under ex vivo confocal microscope in fluorescence mode with acridine orange in a vertical section. Tumor islands (blue circles) are easily recognizable because they are brighter (fluorescent) than the surrounding tissue. [Copyright: (C)2018 Cinotti et al.]

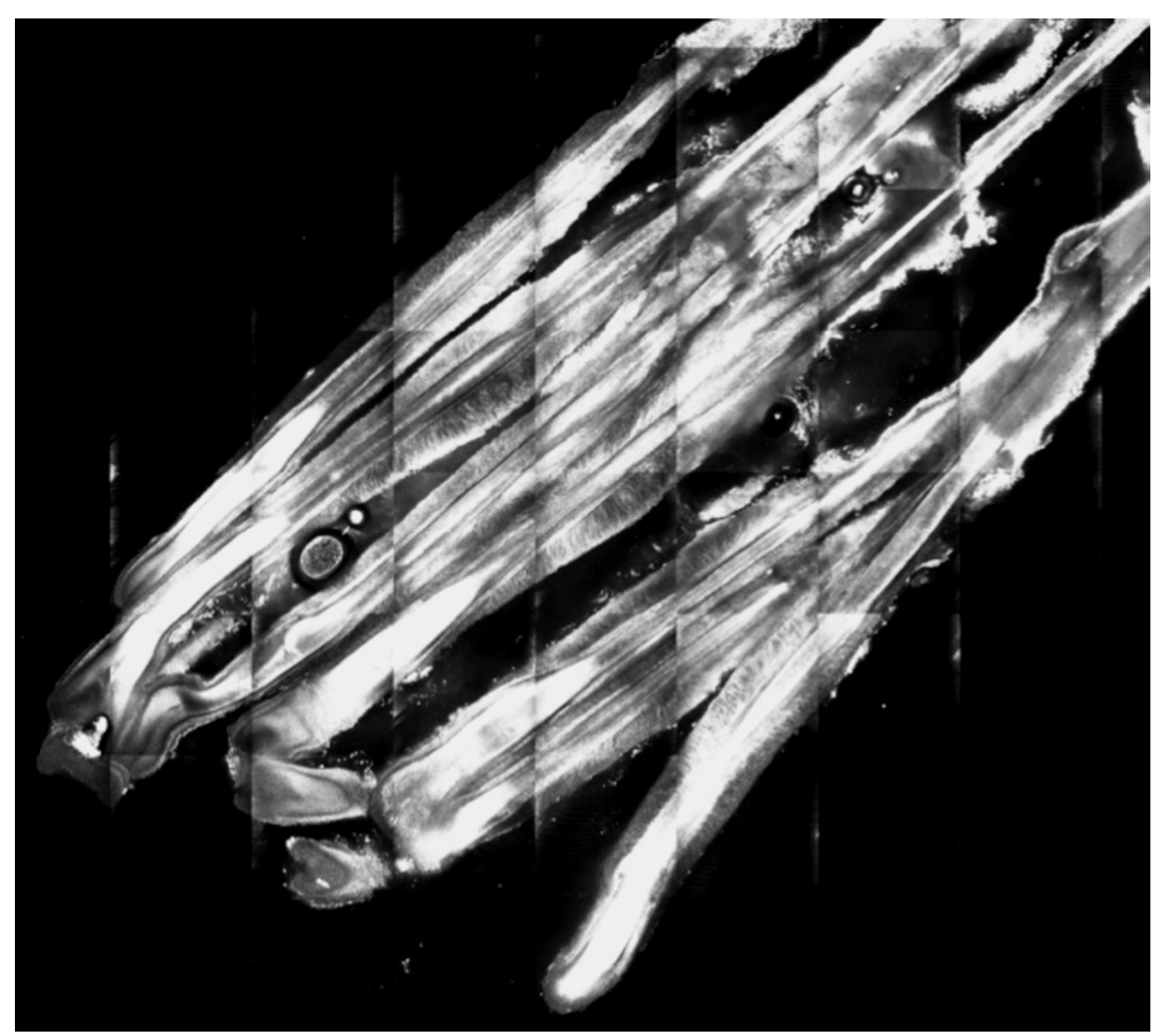

Figure 4. Normal hair shafts under ex vivo reflectance confocal microscopy. [Copyright: (C)2018 Cinotti et al.] 


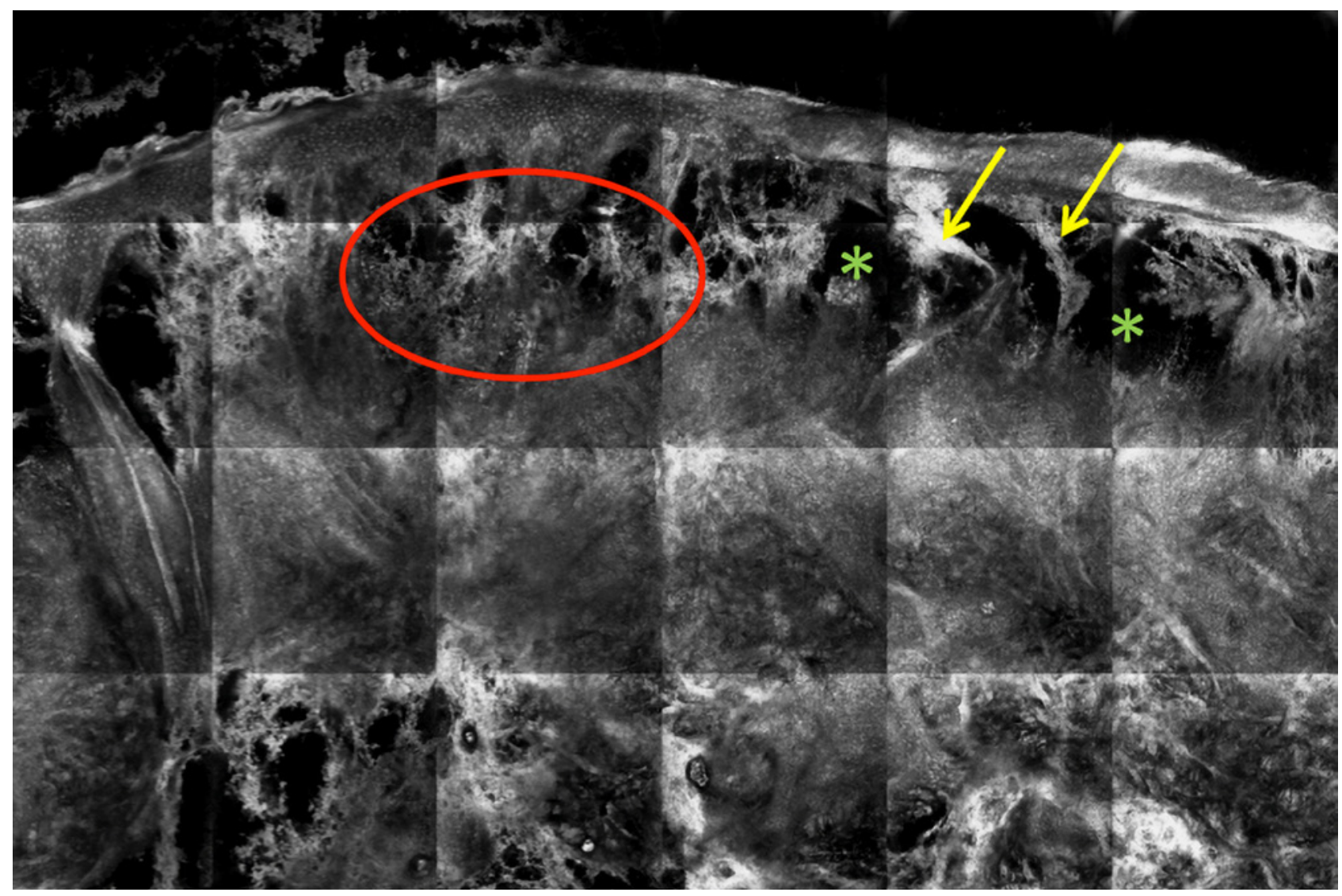

Figure 5. Ex vivo reflectance confocal microscopy examination of a skin biopsy from a plaque of Sweet's syndrome. Dense infiltrate of hyper-reflective cells in the upper half of the dermis corresponding to leukocytes (red circle) and severe edema (hypo-reflective areas, green asterisks) of the upper dermis with strands of dermal collagen stretched across the pseudobullous spaces (yellow arrows) are visible. [Copyright: @2018 Cinotti et al.]

eosin for cellular cytoplasm and dermis) and appears purple and pink [1]. In the future, digital staining could be used in order to change the grayscale into a color scale that mimics the appearance in histology [1].

\section{Ex vivo Confocal Microscopy for the Control of Surgical Margins of Cutaneous Tumors}

Different from MMS, EVCM enables the evaluation of the resection margins immediately after excision without freezing the specimen, thereby reducing the investment of time by two-thirds [10]. To date the analysis of surgical margins has been carried out by cutting the deep and lateral margins ("Tübingen torte" [26]) and analyzing them in vertical plane, perpendicular to the surface of the skin, as is done in conventional histology. Our group proposed an analysis of the entire specimen in horizontal plane, parallel to the surface of the skin ("en face" technique), without cutting the lateral and deep margins [8]. The "en face" technique provides images similar to in vivo reflectance confocal microscopy and is particularly suitable for horizontal spreading tumors [8]. (Figure 6).

Most of the EVCM studies were conducted on BCCs [3-5,7,10,27-31] (Figure 3). Subsequently, EVCM has been proposed for other epithelial tumors [32,33], melanocytic tumors [6,17,34,35] (Figure 7), and Paget's disease [36] (Figure 8).

The overall sensitivity and specificity of fluorescencemode EVCM for detecting BCC with narrow or incomplete margins were $88-96.6 \%$ and $89.2-99 \%$ respectively $[4,7,10,27,30]$. The largest study performed by Bennàssar and colleagues on 80 carcinomas demonstrated an overall sensitivity and specificity for residual BCC of $88 \%$ and $99 \%$ respectively [10].

Possible false positive results can be caused by the presence of hair follicles and eccrine glands or sebaceous glands that may be confused with BCC islands. However, the former reveals no palisading, less fluorescence, and smaller nuclei. Furthermore, it could be difficult to distinguish the infiltrative cords of BCC from the surrounding peritumoral stroma, although the latter show no tendency to cluster.

A few case series reported the use of EVCM for controlling the surgical margins of SCC. Concerning the reflectance mode, an initial study found a positive correlation with histopathology in 13 out of 23 SCCs [11], whereas a sensitivity of $95 \%$ and a specificity of $96 \%$ for the identification of SCC were achieved in a second study on 10 lesions [37]. Only one study has been performed in the fluorescence mode, and 


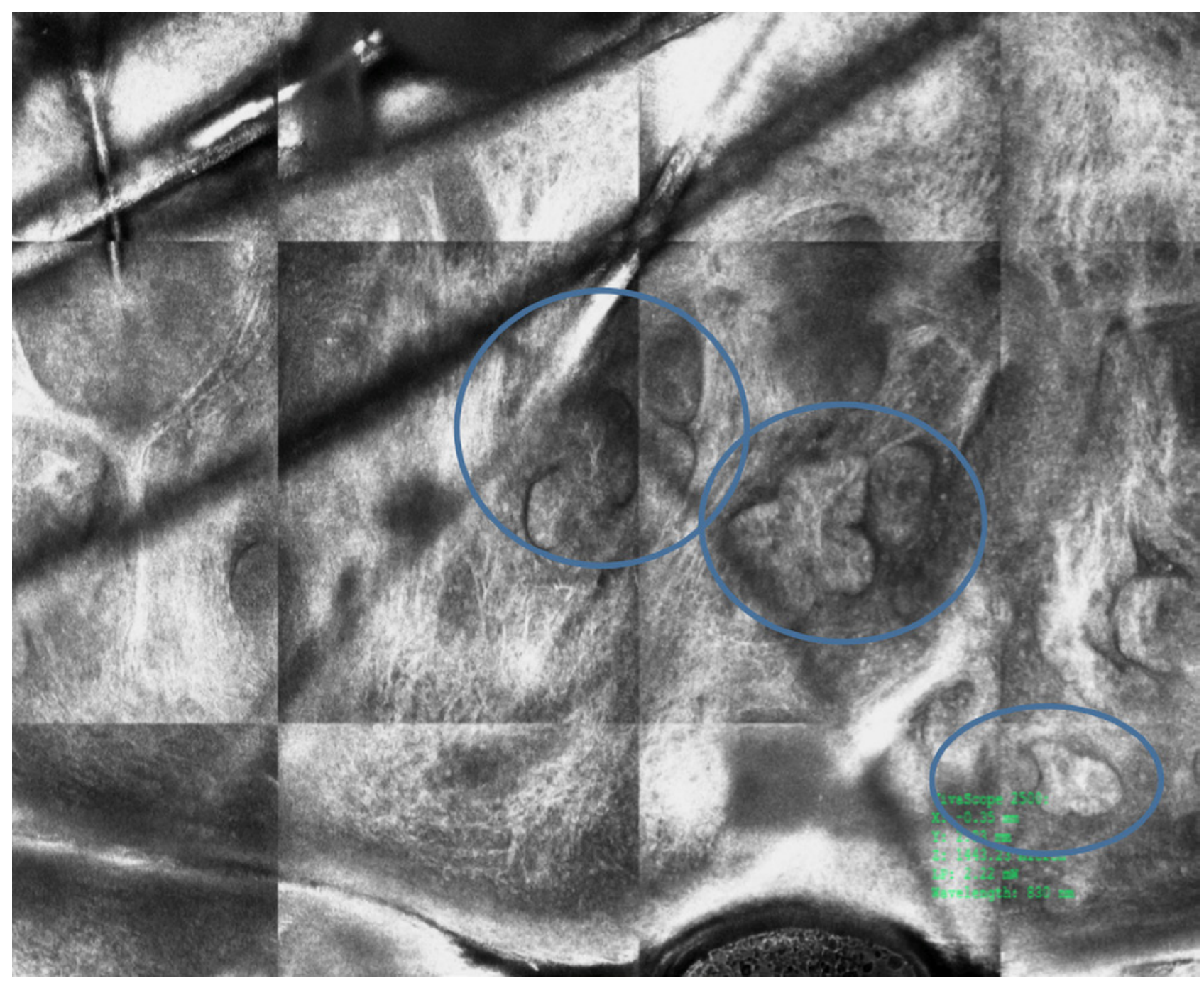

Figure 6. Superficial basal cell carcinoma of the eyelid under ex vivo reflectance confocal microscope and horizontal section. Tumor islands (blue circles) are hyper-reflective and separated from the stroma by clefting. [Copyright: @2018 Cinotti et al.]

EVCM agreed with histopathology in 41 out of 43 mosaics obtained from 34 tumor margins of 13 SCCs [32].

False negative results can be caused by incorrect imaging of the sample due to the presence of artifacts [32] and the inability to properly flatten the fresh tissue [9]. Moreover, a discrepancy with histology can arise when preparing the specimen for standard histological examination and lateral slices are removed from the paraffin-embedded specimen before obtaining definitive sections. The material that is removed could contain tumor tissue seen under EVCM and not under histology.

\section{Basal Cell Carcinoma}

In reflectance mode, large BCCs are easily detected [8]. However, tiny strands of micronodular infiltrating BCCs could remain hidden because of the hyper-reflectivity of the surrounding normal dermis [10].

In fluorescence mode with a contrast agent that stains nuclei, such as acridine orange, BCC islands are highly fluoresecent and are distinguishable from the surrounding tissue because the endogenous autofluorescence from the dermis is relatively weak (Figure 3).

The reported criteria that enable identification of BCC islands under fluorescence confocal microscopy are [38]:

1. Demarcated fluorescent areas with a higher nuclear density than the surrounding epidermis and adnexal structures (nuclear crowding)

2. Peripheral palisading (peripheral polarized and aligned fluorescent cells)

3. Clefting (black fluorescence-free area around the tumor mass)

4. Nuclear pleomorphism

5. Enlarged nucleus/cytoplasm ratio

6. Modified stroma around the tumor (more densely nucleated dermis with fluorescent dots within a black background, corresponding to inflammatory cells and activated fibroblasts)

BCC subtypes can also be identified [7]. In superficial BCC tumor islands are connected to the epidermis and separated from the dermis by some clefts (Figure 3). In nodular 

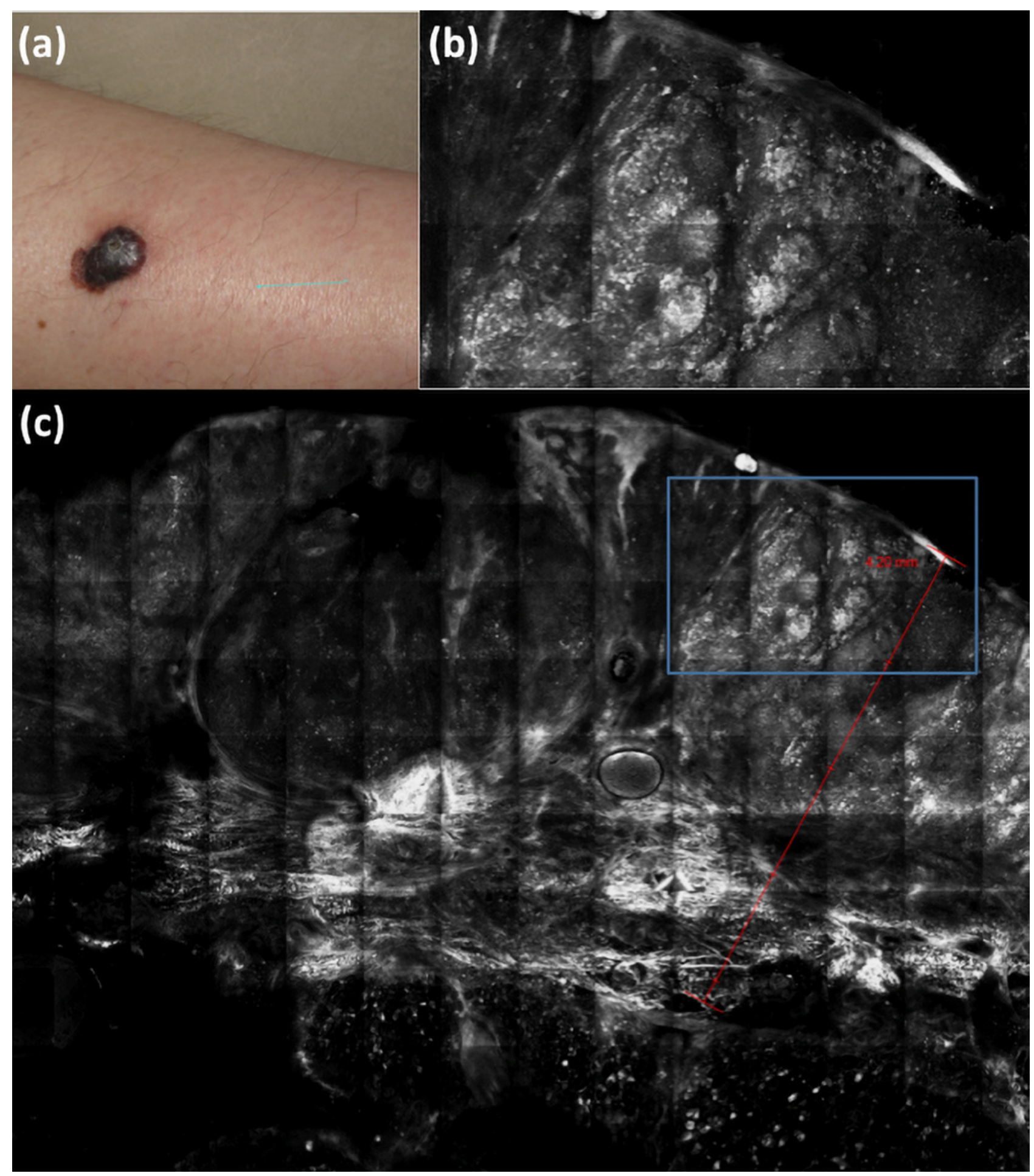

Figure 7. Clinical (a) and ex vivo reflectance confocal microscopy (b, c) aspects of melanoma. Melanoma thickness is marked by the red line (c) and melanoma nests (b, inset in c marked by the blue rectangle) are very easily recognizable because of their brightness (hyper-reflectivity). [Copyright: @2018 Cinotti et al.]

BCC tumor islands are larger and disconnected from the epidermis. In micronodular BCC tumor islands are smaller, and in infiltrative BCC tumor islands are small, elongated, and deep located.

\section{Squamous Cell Carcinoma}

Using the reflectance mode, squamous cell carcinomas (SCC) can be identified by the presence of keratinocytes with densely packed and irregularly distributed nuclei [11,37]. In situ SCC is more difficult to identify because of the difficulty of accurately detecting dyskeratotic cells and nuclear atypia [11,37]. Improved quality of images has been obtained by using acridine orange as fluorochrome [14,32]. A study of 13 SCCs demonstrated that EVCM with the fluorescence mode could also be used to grade this tumor [32]. A well-defined tumor silhouette, numerous keratin pearls, keratin formation, and 


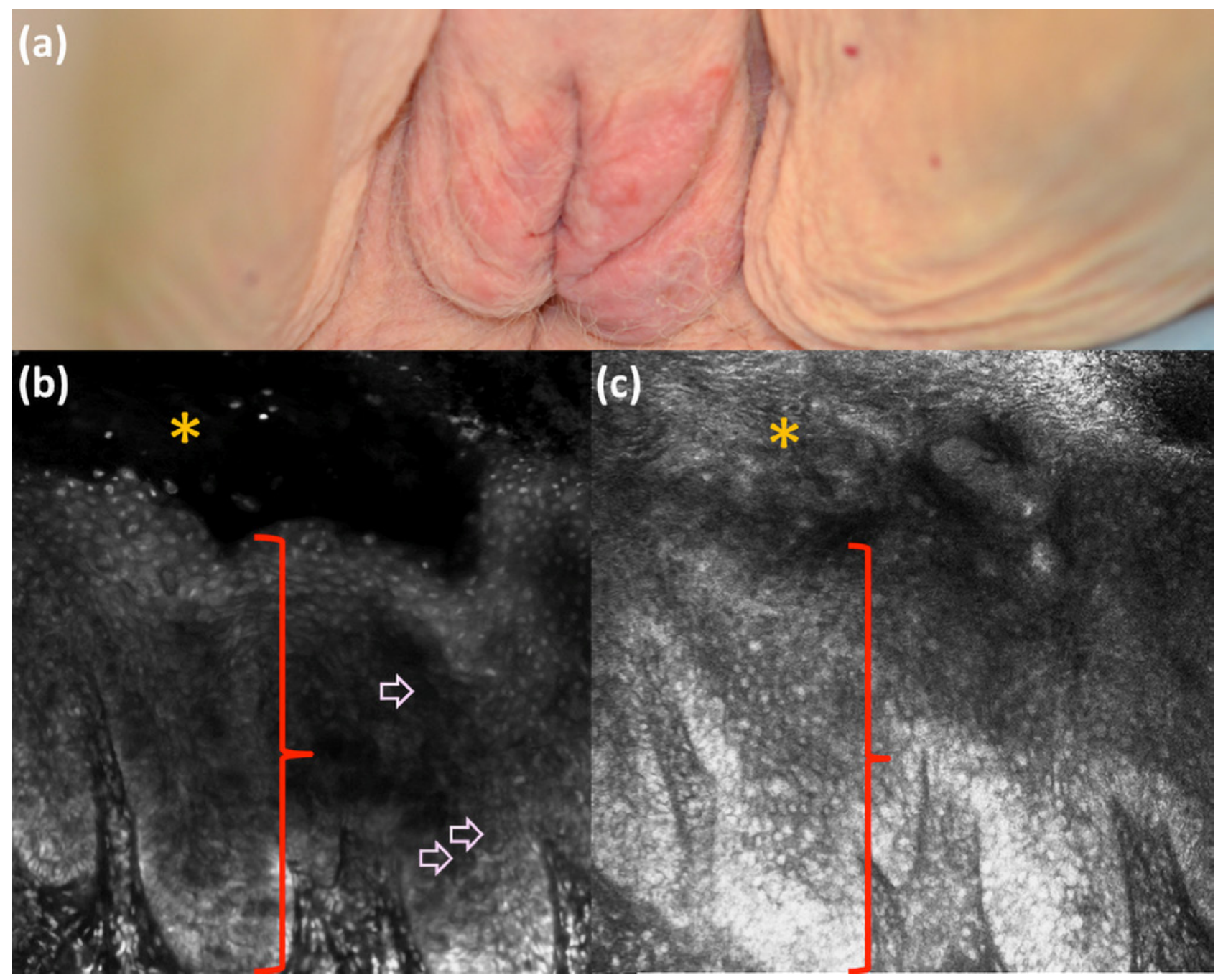

Figure 8. Clinical (a), fluorescence (b), and reflectance (c) ex vivo reflectance confocal microscopy aspect of Paget's disease. Paget's cells in the epidermis (red bracket; stratum corneum is marked by orange star) are visualized better (b, pink arrows) under fluorescence mode with acridine orange than under reflectance mode when comparing images from the same area. [Copyright: @2018 Cinotti et al.]

scarce nuclear pleomorphism correlate with the diagnosis of well-differentiated SCC. Conversely, an ill-defined tumor silhouette, paucity or absence of keratin pearls, and marked nuclear pleomorphism is observed in poorly differentiated tumors. SCCs that are moderately differentiated reveal an intermediate pattern of growth with the presence of keratin formation.

\section{Syringomatous Carcinoma}

EVCM in the fluorescent mode with acridine orange has been performed in only two syringomatous carcinomas [33]. They appeared as highly fluorescent neoplastic cords of monomorphous cells in the dermis.

\section{Melanoma}

Recently, melanoma has been imaged by EVCM [6,17,34]. Proliferation of atypical melanocytes in the epidermis and consumption of the epidermis and nests of atypical melanocytes in the dermis were observed under EVCM in a study of six melanomas with similar features to in vivo reflectance confocal microscopy [6]. A pilot study on 10 melanomas suggested that EVCM could also be used to measure melanoma thickness [34] (Figure 7) with the possible future benefit of being able to choose the correct size of the surgical margins before the surgical excision.

\section{Ex vivo Confocal Microscopy and Nails}

EVCM could be particularly useful for nail tumors in order to confirm intraoperatively the diagnosis of the biopsy specimen and to proceed to the final excision without waiting for the histological examination. Notably, EVCM is more suitable than the optical examination of frozen sections of this site because nail tumors are often very small in size, and it is desirable that no material be wasted in producing sections that subsequently cannot be submitted to conventional pathology.

In a pilot study on six malignant epithelial tumors of the nail apparatus, Debarbieux et al [16] showed that EVCM in fluorescence mode could be a useful tool for the diagnosis 
of invasive SCC, invasive onycholemmal carcinoma, and Bowen's disease showing marked nuclear and cytological atypia and the presence of numerous dyskeratotic cells. In particular, well-demarcated epithelial nests deeply invading the dermis, nuclear pleomorphism (variable size and shape of the nucleus), and densely packed and irregularly organized nuclei have been observed in invasive SCC and onycholemmal carcinoma $[14,39,40]$. Therefore, in these cases it would be possible to perform wide excision of the tumors just after the observation of a biopsy specimen under EVCM, shortening the management. However, in situ SCC and minimally invasive well-differentiated SCC were more difficult to diagnose, showing only focal epithelial acanthosis and not cytological atypia [14].

The same study of Debarbieux et al [14] included three benign epithelial tumors (two onychomatricomas and one onychopapilloma) that were differentiated from SCC because their cellular density was similar to the adjacent nail bed and because the cells had small monomorphic nuclei. Onychomatricomas showed acanthotic and papillomatous epithelium with no identified atypia under EVCM [14]. Interestingly, the dermal papillae embedded within the epithelial proliferation contained numerous spindle cells corresponding to fibroblastas [14]. Onychopapilloma is a very circumscribed epithelial tumor of the nail bed, characterized by thin digitiform epithelial projections within the upper dermis that were visible under EVCM [14]. In our experience, not only can nonpigmented epithelial tumors be recognized under EVCM, but also other amelanotic non-epithelial tumors such as glomus tumor and neurinoma [40].

EVCM seems to be less useful for subungual melanoma because the in vivo device that is used directly on the nail matrix performs well in this special site [15]. Moreover, with EVCM amelanotic melanocytes cannot be distinguished from epithelial cells or dermal inflammatory cells in fluorescence, and their reflectance can be low [14]. Debarbieux et al [41] also used the in vivo device for the ex vivo examination of nail biopsies of pigmented subungual melanoma in a series of eight cases. However, this procedure has limitations in that the specimen is not fixed, tends to move, and is difficult to orient, unlike when using the ex vivo device.

\section{Ex vivo Confocal Microscopy and Infections}

EVCM in reflectance mode has been used to identify the hyphae and/or conidia in onychomycosis [40], tinea capitis [16], and tinea barbae [16]. In reflectance mode hyphae are highly reflective, thick linear structures, and conidia are hyper-reflective roundish bodies with the same aspect of in vivo reflectance confocal microscopy [42-43]. In fluorescence mode with acridine orange, hyphae are bright [22]. Compared to fungal culture, EVCM is faster and does not have the problem of false positive results due to environmental con- tamination because the examination is made concomitantly, just after the specimen collection [22]. EVCM has the great advantage over conventional microscopy in that the sample is left intact with the subsequent possibility of localizing the infectious agent directly in the whole tissue. This could be particularly useful for the fast and precise identification of a fungus in a case of a deep fungal infection such as mucormycosis [22]. To date there is only one study that compares EVCM to conventional microscopy and fungal culture for the identification of dermatophytes, and this study includes only three cases of hair infection [16].

As in vivo confocal microscopy, EVCM can show viral cytopathic effect [20,31]. EVCM has also been used successfully in fluorescence mode with anti-herpes virus simplex 1 (HSV1) antibodies coupled with fluorescein isothiocyanate for the identification of HSV1 from the roof of six vesicles from three different patients [20]. This application is an example of how this device has the potential to identify any pathogen disposing of specific antibodies conjugated with any fluorescent agent that can be excited by a wavelength of $488 \mathrm{~nm}$ or $658 \mathrm{~nm}$. Studies of comparison with standard methods such as Tzanck-test, histopathology, or PCR for HSV infection should be performed.

\section{Conclusions}

In conclusion, EVCM is a relatively new imaging technique that can analyze whole skin samples of up to $2 \mathrm{~cm}$ in diameter without the need to cut it into thin sections. Data for clinical use of EVCM is only available for the intraoperative control of tumor margins in BCC. However, many other tumors are in the evaluation phase, as well as non-tumor diseases such as skin infections. In the future, EVCM could be used in order to quickly obtain architectural and cytological details of any skin sample to orient the clinical diagnosis before definitive histological examination. A promising field seems to be the intraoperative nail evaluation, and a new domain that could be explored is hair shaft examination. At the present time, the main limitation of this technique is the high cost of the device, which limits its widespread use.

\section{References}

1. Bini J, Spain J, Nehal K, Hazelwood V, DiMarzio C, Rajadhyaksha M. Confocal mosaicing microscopy of human skin ex vivo: spectral analysis for digital staining to simulate histology-like appearance. J Biomed Opt. 2011;16:076008.

2. Que SKT. Research techniques made simple: noninvasive imaging technologies for the delineation of basal cell carcinomas. J Invest Dermatol. 2016;136:e33-38.

3. Rajadhyaksha M, Menaker G, Flotte T, Dwyer PJ, González S. Confocal examination of nonmelanoma cancers in thick skin 
excisions to potentially guide mohs micrographic surgery without frozen histopathology. J Invest Dermatol. 2001;117:1137-1143.

4. Karen JK, Gareau DS, Dusza SW, Tudisco M, Rajadhyaksha M, Nehal KS. Detection of basal cell carcinomas in Mohs excisions with fluorescence confocal mosaicing microscopy. Br J Dermatol. 2009;160:1242-1250.

5. Gareau DS, Li Y, Huang B, Eastman Z, Nehal KS, Rajadhyaksha $M$. Confocal mosaicing microscopy in Mohs skin excisions: feasibility of rapid surgical pathology. J Biomed Opt. 2008;13:054001.

6. Hartmann D, Ruini C, Mathemeier L, et al. Identification of ex-vivo confocal laser scanning microscopic features of melanocytic lesions and their histological correlates. J Biophotonics. 2017;10:128-142.

7. Longo C, Rajadhyaksha M, Ragazzi M, et al. Evaluating ex vivo fluorescence confocal microscopy images of basal cell carcinomas in Mohs excised tissue. Br J Dermatol. 2014;171:561-570.

8. Espinasse M, Cinotti E, Grivet D, et al. "En face” ex vivo reflectance confocal microscopy to help the surgery of basal cell carcinoma of the eyelid. Clin Exp Ophthalmol. 2017;45(50):442-447.

9. Cinotti E, Grivet D, Labeille B, et al. The "tissue press": a new device to flatten fresh tissue during ex vivo confocal microscopy examination. Skin Res Technol. 2017;23:121-124.

10. Bennàssar $\mathrm{A}$, Vilata $\mathrm{A}$, Puig $\mathrm{S}$, Malvehy $\mathrm{J}$. Ex vivo fluorescence confocal microscopy for fast evaluation of tumour margins during Mohs surgery. Br J Dermatol. 2014;170:360-365.

11. Chung VQ, Dwyer PJ, Nehal KS, et al. Use of ex vivo confocal scanning laser microscopy during Mohs surgery for nonmelanoma skin cancers. Dermatol Surg. 2004;30:1470-1478.

12. Gerger A, Horn M, Koller S, et al. Confocal examination of untreated fresh specimens from basal cell carcinoma: implications for microscopically guided surgery. Arch Dermatol. 2005;141:1269-1174.

13. Patel YG, Nehal KS, Aranda I, Li Y, Halpern AC, Rajadhyaksha M. Confocal reflectance mosaicing of basal cell carcinomas in Mohs surgical skin excisions. J Biomed Opt. 2007;12:034027.

14. Debarbieux S, Gaspar R, Depaepe L, Dalle S, Balme B, Thomas L. Intraoperative diagnosis of nonpigmented nail tumours with ex vivo fluorescence confocal microscopy: 10 cases. $\mathrm{Br} J$ Dermatol. 2015;172:1037-1044.

15. Fattouh K, Debarbieux S, Depaepe L, Amini-Adle M, Balme $\mathrm{B}$, Thomas L. Routine use of perioperative in vivo reflectance confocal microscopy of the nail matrix in melanonychia striata: about 30 cases. Br J Dermatol. 2017;177:570-573.

16. Cinotti E, Perrot JL, Labeille B, Raberin H, Flori P, Cambazard F. Hair dermatophytosis diagnosed by reflectance confocal microscopy: six cases. J Eur Acad Dermatol Venereol. 2015;29:22572259.

17. Cinotti E, Haouas M, Grivet D, Perrot JL. In vivo and ex vivo confocal microscopy for the management of a melanoma of the eyelid margin. Dermatol Surg. 2015;41:1437-1440.

18. Iovieno A, Longo C, De Luca M, Piana S, Fontana L, Ragazzi M. Fluorescence confocal microscopy for ex vivo diagnosis of conjunctival tumors: a pilot study. Am J Ophthalmol. 2016;168:207216.

19. Cinotti E, Perrot JL, Labeille B, Boukenter A, Ouerdane Y, Cosmo $\mathrm{P}$, et al. Identification of a soft tissue filler by ex vivo confocal microscopy and Raman spectroscopy in a case of adverse reaction to the filler. Skin Res Technol. 2015;21:114-118.

20. Cinotti E, Perrot JL, Labeille B, et al. First identification of the herpes simplex virus by skin-dedicated ex vivo fluorescence confocal microscopy during herpetic skin infections. Clin Exp Dermatol. 2015;40:421-425.

21. Cinotti E, Labeille B, Douchet $C$, et al. Role of in vivo and ex vivo confocal microscopy and of optical coherence tomography as aids in the diagnosis of molluscum contagiosum. Ann Dermatol Venereol. 2016;143:564-566.

22. Leclercq A, Cinotti E, Labeille B, Perrot JL, Cambazard F. Ex vivo confocal microscopy: a new diagnostic technique for mucormycosis. Skin Res Technol. 2016;22:203-207.

23. Forest F, Cinotti E, Yvorel V, et al. Ex vivo confocal microscopy imaging to identify tumor tissue on freshly removed brain sample. J Neurooncol. 2015;124:157-164.

24. Ragazzi M, Piana S, Longo C, et al. Fluorescence confocal microscopy for pathologists. Mod Pathol. 2014;27:460-471.

25. Ragazzi M, Longo C, Piana S. Ex Vivo (Fluorescence) confocal microscopy in surgical pathology: state of the art. Adv Anat Pathol. 2016;23:159-169.

26. Kopke LF, Konz B. Micrographic surgery. A current methodological assessment. Hautarzt. 1995;46:607-614.

27. Gareau DS, Patel YG, Li Y, et al. Confocal mosaicing microscopy in skin excisions: a demonstration of rapid surgical pathology. $J$ Microsc. 2009;233:149-59.

28. Ziefle S, Schüle D, Breuninger H, Schippert W, Moehrle M. Confocal laser scanning microscopy vs 3-dimensional histologic imaging in basal cell carcinoma. Arch Dermatol. 2010;146:843-847.

29. Al-Arashi MY, Salomatina E, Yaroslavsky AN. Multimodal confocal microscopy for diagnosing nonmelanoma skin cancers. Lasers Surg Med. 2007;39:696-705.

30. Larson B, Abeytunge S, Seltzer E, Rajadhyaksha M, Nehal K. Detection of skin cancer margins in Mohs excisions with highspeed strip mosaicing confocal microscopy: a feasibility study. $\mathrm{Br}$ J Dermatol. 2013;169:922-926.

31. Schüle D, Breuninger H, Schippert W, Dietz K, Moehrle M. Confocal laser scanning microscopy in micrographic surgery (threedimensional histology) of basal cell carcinomas. Br J Dermatol. 2009;161:698-700.

32. Longo C, Ragazzi M, Gardini S, et al. Ex vivo fluorescence confocal microscopy in conjunction with Mohs micrographic surgery for cutaneous squamous cell carcinoma. J Am Acad Dermatol. 2015;73:321-322.

33. Longo C, Ragazzi M, Gardini S, Moscarella E, Argenziano G. Ex vivo fluorescence confocal microscopy of eccrine syringomatous carcinoma: a report of 2 cases. JAMA Dermatol. 2015;151:10341036.

34. Hartmann D, Krammer S, Ruini C, Ruzicka T, von Braunmühl T. Correlation of histological and ex-vivo confocal tumor thickness in malignant melanoma. Lasers Med Sci. 2016;31:921-927.

35. Cinotti E, Perrot J-L, Labeille B, et al. Contribution of reflectance confocal microscopy in the diagnosis of eyelid dermal nevus. Ann Dermatol Venereol. 2015;142:226-228.

36. Debarbieux S, Dalle S, Depaepe L, Jeanniot PY, Poulalhon N, Thomas L. Extramammary Paget's disease of the scalp: examination by in vivo and ex vivo reflectance confocal microscopy. Skin Res Technol. 2014;20:124-126.

37. Horn M, Gerger A, Koller S, et al. The use of confocal laserscanning microscopy in microsurgery for invasive squamous cell carcinoma. Br J Dermatol. 2007;156:81-84.

38. Bennàssar A, Carrera $\mathrm{C}$, Puig S, Vilalta $\mathrm{A}$, Malvehy J. Fast evaluation of 69 basal cell carcinomas with ex vivo fluorescence confocal microscopy: criteria description, histopathological 
correlation, and interobserver agreement. JAMA Dermatol. 2013;149:839-847.

39. Fernandes Massa A, Debarbieux S, Depaepe L, Dalle S, Balme B, Thomas L. Pigmented squamous cell carcinoma of the nail bed presenting as a melanonychia striata: diagnosis by perioperative reflectance confocal microscopy. Br J Dermatol. 2013;169:198199.

40. Cinotti E, Fouilloux B, Perrot JL, Labeille B, Douchet C, Cambazard F. Confocal microscopy for healthy and pathological nail. $J$ Eur Acad Dermatol Venereol. 2014;28:853-858.
41. Debarbieux S, Hospod V, Depaepe L, Balme B, Poulalhon N, Thomas L. Perioperative confocal microscopy of the nail matrix in the management of in situ or minimally invasive subungual melanomas. Br J Dermatol. 2012;167:828-836.

42. Forest F, Cinotti E, Habougit C, et al. Rapid characterization of human brain aspergillosis by confocal microscopy on a thick squash preparation. Cytopathology. 2016;27:221-222.

43. Cinotti E, Perrot JL, Labeille B, Cambazard F. Reflectance confocal microscopy for cutaneous infections and infestations. J Eur Acad Dermatol Venereol. 2016;30:754-763. 